\title{
Fitness differences and persistent founder effects determine the clonal composition during population build-up in Daphnia
}

\author{
Anne Thielsch, Nicole Glass, Bruno Streit, Luc De Meester, Raquel Ortells and Klaus Schwenk \\ A. Thielsch (thielsch@uni-landau.de) and K. Schwenk, Inst. for Environmental Science, Molecular Ecology, Univ. of Koblenz-Landau, \\ Fortstraße 7, DE-76829 Landau, Germany. - N. Glass and B. Streit, Dept of Ecology and Evolution, Goethe-Univ. - Biologicum, \\ Max-von-Laue-Straße 13, DE-60438 Frankfurt am Main, Germany. - L. De Meester, Laboratory Aquatic Ecology, Evolution and \\ Conservation, KU Leuven - Univ. of Leuven, Charles Deberiotstraat 32, BE-3000 Leuven, Belgium. - R. Ortells, Inst. Cavanilles de \\ Biodiversitat i Biologia Evolutiva, Univ. of Valencia, C/Catedrático José Beltrán, 2, ES-46980 Paterna, Spain.
}

\begin{abstract}
During the initial stages of population build-up in new habitats, the time at which immigrants arrive may influence their contribution to the population and thus determine population genetic structure. While the numerical advantage associated with founder effects may promote dominance of the offspring of early colonizers, fitness differences associated with ecological differences among genotypes may potentially overwhelm these founder effects. We conducted an outdoor mesocosm experiment to test whether the sequence of arrival determines the relative contribution of genetic lineages (clones) to populations of the water flea Daphnia. A set of D. galeata clones was inoculated pairwise in different time sequences and clonal abundances were monitored over time. In general, we found that, after six weeks (four generations), clones with an initial time advantage of five or fifteen days (1/3 of a generation or one generation) had higher relative abundances than in the treatments with simultaneous inoculations. These differences were in some cases maintained over a longer period (nine months). However, in cases where abundances of genetic lineages varied strongly when inoculated simultaneously, reflecting strong differences in fitness among clones in our experimental containers, initial numerical advantages could be overwhelmed and the competitively strongest clone became dominant after nine months independently of inoculation sequence. Our results highlight the importance of two different processes that determine the fate of clonal lineages and ultimately determine population genetic structure. First, the sequence of arrival is crucial, with an advantage of five days being already sufficient to dominate the population in specific cases. Second, if fitness differences among clones are sufficiently strong, then they may overrule founder effects.
\end{abstract}

A fundamental knowledge of natural populations and how they establish is crucial in evolutionary and ecological research. Additionally, in times of severe global change an understanding of population assembly is essential to properly evaluate consequences of, e.g. habitat fragmentation, habitat alteration, and invasion of alien species, as those may necessitate recolonization of impacted habitats. The build-up of new populations, i.e. the establishment of individuals of a certain species in a given habitat over time, is impacted by at least two different processes. First, colonizing individuals are likely to vary in response to local environmental conditions (for example in the aphid Rhopalosiphum padi: Bieri et al. 2009, the rotifer Brachionus plicatilis: Campillo et al. 2009, or the crustacean Daphnia galeata: Macháček and Sed'a 2007) and thus display differences in fitness. Natural selection will then result in a differential contribution of immigrants to the population. Second, the chronology in which individuals arrive at a habitat may influence population genetic structure because early colonists may profit from a numerical advantage. As individuals arrive, they consume resources and grow, modifying the habitat such that establishment success and subsequent growth of individuals arriving later is reduced (persistent founder effect: Boileau et al. 1992, also known as high-density blocking: Hewitt and Ibrahim 2001, at the community level this process is called priority effect: Morin 1999). If the early colonists are given sufficient time, founder effects may be enhanced as the resident population genetically adapts to local environmental conditions (De Meester et al. 2002).

Lentic freshwater habitats are ideal systems to study population assembly as they are limited in size, exhibit a specific history of succession, and are colonized very quickly (Louette and De Meester 2005). Because many freshwater species possess high potential for passive dispersal as they produce so-called dormant eggs that withstand cold, heat and desiccation (Brendonck and De Meester 2003), the arrival of new genotypes to a population is very likely. Short-distance dispersal of adult zooplankton has also been reported and is mostly carried out by animal vectors (Allen 2007). The water flea Daphnia is an intensively studied model organism in freshwater ecosystems. Daphnia individuals reproduce via cyclic parthenogenesis (Zaffagnini 1987) enabling them to 
reproduce clonally in a fast and efficient way if environmental conditions are favourable, and to reproduce sexually if conditions deteriorate, resulting in the production of dormant eggs. This reproductive mode may potentially enhance founder effects as the carrying capacity is reached very quickly due to clonality, while sexual recombination enables local adaptation more swiftly (De Meester et al. 2002).

The main aim of our study was to investigate the impact of founder effects on the genetic structure of newly established populations. Therefore, we conducted a colonization experiment in outdoor mesocosms using the zooplankter Daphnia galeata. We manipulated the time of invasion for several pairs of genotypes and determined whether the relative abundances of the resulting genotypes were more determined by the differences in colonization time or by genotypic differences in performance. Further, we wanted to assess the effect of the duration of the time lag on founder effects, and to what extent these effects remain stable over a longer time period.

\section{Methods}

\section{Collection of clones}

Daphnia galeata clones were selected from three different sampling sites in Belgium. Six of the eight clones (genotypes BE03, 04, 05, 10, 11 and 15) were sampled near Beringen $\left(51^{\circ} 00^{\prime} 18^{\prime \prime} \mathrm{N}, 5^{\circ} 18^{\prime} 17^{\prime \prime} \mathrm{E}\right)$ and one was sampled from a lake near Maaseik ( $51^{\circ} 05^{\prime} 49^{\prime \prime} \mathrm{N}, 5^{\circ} 48^{\prime} 16^{\prime \prime} \mathrm{E}$; genotype MA12). Samples were collected on 9 May 2007 using a plankton net with a mesh size of $200 \mu \mathrm{m}$ and individuals were isolated to establish clonal cultures in the laboratory. One additional clone (HE01) was hatched from sediments from a pond near Oud Heverlee $\left(51^{\circ} 21^{\prime} 01.97^{\prime \prime} \mathrm{N}, 3^{\circ} 19^{\prime} 49.58^{\prime \prime} \mathrm{E}\right)$. The sediments of this pond were sampled in October 2006 and stored at $4^{\circ} \mathrm{C}$. All experimental clones were raised in the laboratory under standardized conditions for at least two months prior to the start of the experiment to reduce maternal effects. They were cultured in ADaM (Klüttgen et al. 1994) with a light:dark cycle of 16:8 h, at around $18-20^{\circ} \mathrm{C}$ and under optimal food conditions ( $1 \mathrm{mg}$ carbon $1^{-1}$ Scenedesmus obliquus).

\section{Molecular identification of clones}

DNA preparation was conducted using proteinase $\mathrm{K}$ digestion according to Schwenk et al. (1998). Prior to DNA preparation, ethanol preserved individuals were incubated for $4-16 \mathrm{~h}$ at $4^{\circ} \mathrm{C}$ in $1 \mathrm{ml} \mathrm{TE}$ buffer $(1 \mathrm{mM}$ Tris, $0.1 \mathrm{mM}$ EDTA, $\mathrm{pH}$ 8.0) to remove the ethanol. Further, we amplified twelve microsatellite loci (Dp281NB, SwiD14, DaB10/14, DaB17/17, Dp196NB, SwiD6, SwiD12, SwiD18, Dgm105, Dgm109, Dgm112 and Dp519; Brede et al. 2006) for several individuals of each clone according to the protocols published by Thielsch et al. (2009). Based on the result of this initial screening, we selected three discriminating loci (SwiD12, SwiD14 and SwiD18) that were sufficiently variable to differentiate the genotypes. Polymerase chain reactions for the discriminating loci were performed in $0.2 \mathrm{ml}$ tubes with $10 \mu \mathrm{l}$ reaction volume containing
$2.4 \mathrm{mM} \mathrm{MgCl} 2,1 \times$ PCR buffer, $0.25 \mathrm{mM}$ of each dNTP, $0.2 \mu \mathrm{M}$ (SwiD14 and SwiD18) or $0.1 \mu \mathrm{M}$ (SwiD12) of forward and reverse primer, $0.5 \mathrm{U}$ (SwiD12 and SwiD14) or $1 \mathrm{U}$ (SwiD18) Taq polymerase, $0.2 \mathrm{mg} \mathrm{ml}^{-1}$ (for loci SwiD14 and SwiD18) or $0.1 \mathrm{mg} \mathrm{m}^{-1}$ (for locus SwiD12) BSA, 8\% DMSO for locus SwiD18 and 2-4 $\mu$ l prepared DNA. Forward primers were labelled with the fluorescent dyes Alexa 647 (Invitrogen; SwiD14) and IRD700 (MWG; SwiD12 and SwiD18). Cycling conditions for PCR started with a 3 min denaturing step at $95^{\circ} \mathrm{C}$ followed by 35 cycles $(26$ cycles for locus SwiD14) of $1 \mathrm{~min}$ steps at $95^{\circ} \mathrm{C}$, at $55^{\circ} \mathrm{C}(\mathrm{SwiD} 12$, SwiD18) or $60^{\circ} \mathrm{C}(\mathrm{SwiD} 14)$ and $72^{\circ} \mathrm{C}$. A final 7 min synthesis step at $72^{\circ} \mathrm{C}$ completed all programs. Amplicons were diluted and electrophoresed on a CEQ 2000 (denaturation at $90^{\circ} \mathrm{C}$ for $2 \mathrm{~min}$; injection at $2.0 \mathrm{kV}$ for $30 \mathrm{~s}$; separation at $6.0 \mathrm{kV}$ for $45 \mathrm{~min}$ ) with a self-designed size standard based on Lambda phage DNA (Symonds and Lloyd 2004).

\section{Mesocosm experiment}

The experiment was performed in the Aquatic Research Experimental Area (ARENA) at KU Leuven (Heverlee, Belgium), using white conical plastic buckets (diameter ${ }_{\text {bottom }}$ $410 \mathrm{~mm}$, diameter top $480 \mathrm{~mm}$, height $645 \mathrm{~mm}$, volume 100 l) filled with $80 \mathrm{l}$ of tap water. Populations were established using four combinations of clonal pairs. Two combinations included clones from the same habitat (BE04/BE10 and BE05/BE11) while the other two combination consisted of clones from different habitats (HE01/BE03 and BE15/ MA12).

Using eight different clones in four pair-wise combinations allowed us to test for differences in fitness and persistent founder effects. Clones were either inoculated at the same time, or with a 5 or 15 days time lag (two inoculation events). The latter treatments were carried out reciprocally, giving one or the other clone a time advantage. The treatment in which clones were inoculated at the same time directly assessed fitness differences between clones. Each treatment was replicated three times. The design resulted in four clone combinations $\times 5$ inoculation treatments $\times 3$ replicates $=$ 60 experimental buckets. Treatments were randomly assigned to buckets. Buckets were covered with mosquito net to avoid contamination, e.g. dispersal by aquatic insects. Twenty-four hours after filling the buckets with water, they received algae as food source. The algae stock was grown from a diverse phytoplankton community belonging to two ponds nearby the experimental site in May 2007. Water was first filtered through a $30 \mu \mathrm{m}$ net to remove zooplankton and then forty litres of this phytoplankton suspension were added to a large container with $560 \mathrm{l}$ of tap water containing nutrients $(3.48 \mathrm{~g}$ $\mathrm{KH}_{2} \mathrm{PO}_{4}$ and $34.8 \mathrm{~g} \mathrm{NaNO}_{3}$ ). In order to monitor algae growth, the concentration of chlorophyll a was determined in the laboratory every three to four days by measuring absorbance at $665 \mathrm{~nm}$ with a fluorimeter. The algae community was allowed to grow approximately 50 days before a homogenous inoculum of 41 was added to each experimental bucket.

The experiment started on 9 July 2007, although the initial inoculation of founder genotypes was done on different days for practical reasons (Supplementary material Appendix 1 Table A1). We used 50 individuals per clone per inoculation (Supplementary material Appendix 1 Table A1). During the 
experiment chlorophyll a content $\left(\mathrm{chl}_{\mathrm{a}}\right)\left[\mu \mathrm{g} \mathrm{l}^{-1}\right]$, temperature $\left[{ }^{\circ} \mathrm{C}\right]$, oxygen content $\left[\mathrm{mg} \mathrm{l}^{-1}\right]$, conductivity $\left[\mu \mathrm{S} \mathrm{cm}^{-1}\right]$ and $\mathrm{pH}$ were monitored every two weeks in the first phase of the experiment (until the first sampling, after six weeks) and two more times later on (November 2007 and May 2008).

The first sampling campaign took place approximately six weeks after the start of the experiment (September 2007; Supplementary material Appendix 1 Table A1). We sampled $20 \%$ of the volume of the experimental bucket with a tubesampler, which allowed us to sample the whole water column. The zooplankton was filtered over a $64 \mu \mathrm{m}$ plankton gauze. All Daphnia individuals were counted and population size was extrapolated. If there were less than 50 individuals in the initial sample, we sampled again shortly afterwards (10 out of 60 populations). In addition, the occurrence of other mesozooplankton taxa was monitored, individuals of different taxonomic groups were counted and the population size was extrapolated for each group as well.

After the first sampling campaign, we left the buckets in the experimental field for a period of approximately seven more months, including a winter period, until May 2008. This resulted in a total experimental period of nine months. We then sampled all populations by emptying the buckets and taking all available plankton for genetic identification and population size estimation. On each sampling date, 30 D. galeata individuals (when available) were collected from each container and their genotypes screened.

\section{Statistical tests for fitness and founder effects}

Bucket 4 was not included in the analysis of the six-week samples as we found less than 30 individuals even after sampling twice. In addition, buckets 29 and 30 were excluded completely as not enough juveniles were available for the second inoculation. Besides buckets 29 and 30, we had to exclude 19 more buckets of the nine-month samples as we did not retrieve 30 individuals from these experimental units. All statistical analyses were conducted without these buckets (six weeks: 57 experimental units analysed; nine months: 39 experimental units analysed; Table 1). Genotype abundances of all genotype combinations (HE01/BE03, BE04/BE10, BE05/BE11 and BE15/MA12), inoculation order (0 days, 5 days and 15 days advantage), and sampling times (six weeks or nine months) were visualized as box plots. Fitness differences among genotypes (treatment 0 days, sampling after six weeks) were tested for using $G$ statistics implemented in PopTools ver. 3.1 (Hood 2009). To describe and analyse the impact of several parameters (inoculation order, sampling time, population size, genotype identity, conductivity, oxygen content, chlorophyll a content, $\mathrm{pH}$, duration of the experiment, inoculation date, occurrence and abundance of other mesozooplankton taxa) on the detected genotype abundances, we used partial least squares regressions (PLS regression) implemented in The Unscrambler X ver. 10.0.1. All values were standardized and significance was estimated by uncertainty tests with full cross-validation before analyses. PLS regression combines features from principal component analyses and multiple regressions and aims to predict one dependent variable (in our case genotype abundances) from several explanatory variables (e.g. inoculation order, sampling time, population size, genotype identity; Abdi 2007). This method avoids multicollinearity as the variance of a multivariate dataset is transferred to reduced principal components (Wold 1975, Wold et al. 2001). Another big advantage

Table 1. Relative genotype abundances of 30 individuals selected from each container during the first and the second sampling. Founder $=\mathrm{clone}$ that was inoculated first; $\Delta t=$ time advantage of the founder clone in days until the second clone was inoculated; 6 weeks =abundance of founder genotype in percent after six weeks of experimental time; 9 months = abundance of founder genotype in percent after nine months; $\mathrm{NA}=$ no data available.

\begin{tabular}{|c|c|c|c|c|c|c|c|c|c|c|}
\hline \multirow[b]{2}{*}{ Combination } & \multirow[b]{2}{*}{ Founder } & \multirow[b]{2}{*}{$\Delta t[\mathrm{~d}]$} & \multicolumn{4}{|c|}{6 weeks } & \multicolumn{4}{|c|}{9 months } \\
\hline & & & Replicate 1 & Replicate 2 & Replicate 3 & Mean & Replicate 1 & Replicate 2 & Replicate 3 & Mean \\
\hline \multirow[t]{6}{*}{ BE03-HE01 } & BE03 & 0 & 43.3 & 53.3 & 43.3 & 46.6 & $\mathrm{NA}$ & 0.0 & 33.3 & 16.7 \\
\hline & BE03 & 5 & 70.0 & 33.3 & 40.0 & 47.8 & 80.0 & 93.3 & 10.0 & 61.1 \\
\hline & BE03 & 15 & 76.7 & 73.3 & 56.7 & 68.9 & 56.7 & 13.3 & NA & 35.0 \\
\hline & HE01 & 0 & 56.7 & 46.7 & 56.7 & 53.4 & NA & 100 & 66.6 & 83.3 \\
\hline & HE01 & 5 & NA & 26.7 & 16.7 & 21.7 & 93.3 & 33.3 & 100 & 75.5 \\
\hline & HE01 & 15 & 86.7 & 90.0 & 93.3 & 90.0 & 86.7 & NA & NA & NA \\
\hline \multirow[t]{6}{*}{ BE04-BE10 } & BE04 & 0 & 3.3 & 20.0 & 36.7 & 20.0 & 0.0 & 0.0 & 0.0 & 0.0 \\
\hline & BE04 & 5 & 13.3 & 0.0 & 0.0 & 4.4 & 0.0 & 0.0 & 0.0 & 0.0 \\
\hline & BE04 & 15 & 50.0 & 26.7 & 6.7 & 27.8 & NA & 0.0 & NA & NA \\
\hline & BE10 & 0 & 96.7 & 80.0 & 63.3 & 80.0 & 100 & 100 & 100 & 100 \\
\hline & BE10 & 5 & 100 & 73.3 & 80.0 & 84.4 & 100 & 100 & 100 & 100 \\
\hline & BE10 & 15 & 100 & NA & NA & NA & 100 & NA & NA & NA \\
\hline \multirow[t]{6}{*}{ BE05-BE11 } & BE05 & 0 & 46.7 & 53.3 & 33.3 & 44.4 & NA & NA & 50.0 & NA \\
\hline & BE05 & 5 & 56.7 & 53.3 & 36.7 & 48.9 & NA & NA & NA & NA \\
\hline & BE05 & 15 & 86.7 & 96.7 & 96.7 & 93.4 & NA & NA & 93.3 & NA \\
\hline & BE11 & 0 & 53.3 & 46.7 & 66.7 & 55.6 & NA & NA & 50.0 & NA \\
\hline & BE11 & 5 & 100 & 86.7 & 96.7 & 94.5 & 90.0 & 96.7 & NA & 93.4 \\
\hline & BE11 & 15 & 90.0 & 80.0 & 96.7 & 88.9 & NA & NA & 100 & NA \\
\hline \multirow[t]{6}{*}{ BE15-MA12 } & BE15 & 0 & 100 & 86.7 & 83.3 & 90.0 & 100 & 100 & 100 & 100 \\
\hline & BE15 & 5 & 100 & 90.0 & 96.7 & 95.6 & NA & 100 & 100 & 100 \\
\hline & BE15 & 15 & 100 & 100 & 100 & 100 & 100 & 100 & NA & 100 \\
\hline & MA12 & 0 & 0.0 & 13.3 & 16.7 & 10.0 & 0.0 & 0.0 & 0.0 & 0.0 \\
\hline & MA12 & 5 & 60.0 & 20.0 & 70.0 & 50.0 & NA & 0.0 & 0.0 & 0.0 \\
\hline & MA12 & 15 & 43.3 & 73.3 & 86.7 & 67.8 & 0.0 & 0.0 & 0.0 & 0.0 \\
\hline
\end{tabular}


is the possibility to use qualitative and quantitative data in the creation of regression equations. In addition, PLS regression is reliable in identifying relevant variables, especially in cases of small sample sizes (Carrascal et al. 2009).

\section{Results}

Environmental parameters ( $\mathrm{chl}_{\mathrm{a}}$, temperature, oxygen, $\mathrm{pH}$ and conductivity; Supplementary material Appendix 1 Table A2-A6), community structure (occurrence and abundance of other mesozooplankton taxa; Supplementary material Appendix 1 Table A7), and population size of Daphnia galeata (Supplementary material Appendix 1 Table A8) were monitored in each bucket, but no significant impact on genotype abundance was observed (Fig. 1). After nine months, evidence for sexual reproduction was recorded, i.e. resting stages and recombinant genotypes, but were not further considered due to their low frequencies.

When clones were inoculated simultaneously, they showed variable differences in competitive strength (Table 1). In two combinations we detected initial genotype abundances that did not differ significantly among genotypes after six weeks experimental time (BE03-HE01: $\mathrm{G}=0.79, \mathrm{p}=0.68$ ); BE05-BE11: $G=2.50, p=0.29)$. The other two genotype combinations (BE04-BE10: $\mathrm{G}=12.89$, $\mathrm{p}<0.01$; BE15MA12: $G=16.24, p<0.001)$ showed one genotype that was competitively superior throughout the whole experiment, BE10 and BE15, respectively.

Although strong competitive differences among clones were only detected in two genotype combinations the overall influence of genotype fitness on genotype abundances was very high as indicated by multivariate PLS regression (Fig. 1, 2 ). Besides individual fitness of genotypes, inoculation order
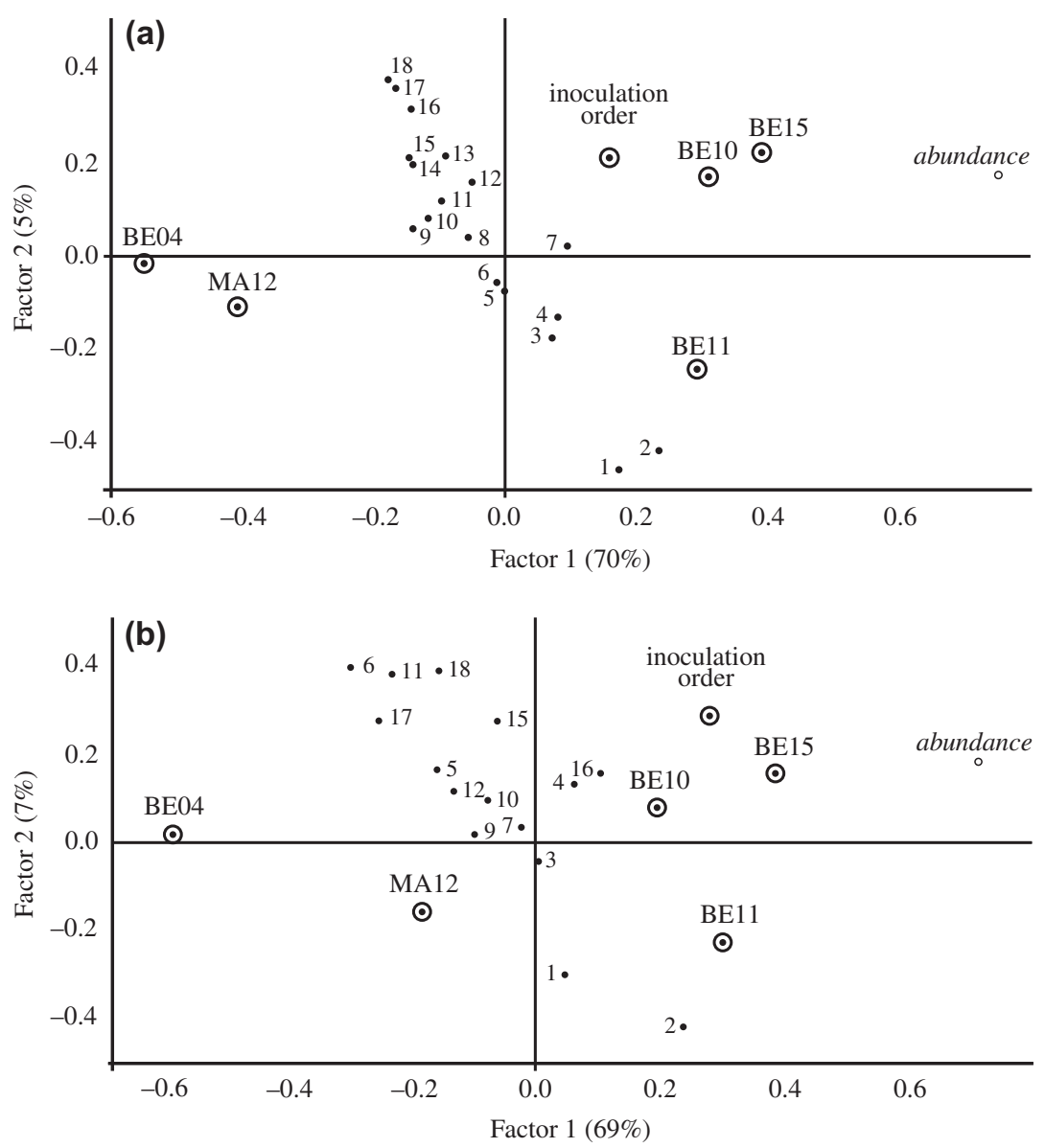

Figure 1. PLS regression plots of the two principal components with $\mathrm{x}$ and $\mathrm{y}$ loadings. (a) data of both sampling events (six weeks and nine months) are included; Factor 1 and 2 explain $70 \%$ and $5 \%$ of the genotype abundance; full cross validation: $\mathrm{R}^{2}=0.66, \mathrm{RSME}=22.08$. (b) only data of the sampling after six weeks are included; Factor 1 and 2 explain $69 \%$ and $7 \%$ of the genotype abundance; full cross validation: $\mathrm{R}^{2}=0.64, \mathrm{RSME}=18.83$. The $\mathrm{y}$-variable 'genotype abundance' is shown as small open circle and in italic lettering in both plots, in contrast $\mathrm{x}$-variables are shown as black filled circles and significant $\mathrm{x}$-variables (genotype identity: BE04, BE10, BE11, BE15, MA12; and inoculation order) are marked with additional circles. Along the $\mathrm{x}$-axis (Factor 1), positive variables indicate a positive impact on genotype abundance and the closer the variables are to abundance, the higher is the impact on the y (dependent) variable. In reverse, negative variables indicate a negative impact on the y-variable genotype abundance. Non-significant variables are abbreviated with numbers from 1-18: 1 = inoculation date, $2=$ conductivity, $3=$ genotype BE11, $4=$ abundance of Acanthocyclops, $5=$ occurrence of Daphnia magna, $6=$ population size, 7 = genotype HE01, $8=$ abundance of Chydorus, $9=$ chlorophyll a content, $10=$ genotype BE03, $11=$ abundance of Daphnia magna, $12=\mathrm{pH}, 13=$ occurrence of Chydorus, $14=$ sampling time, $15=$ duration of the experiment, $16=$ abundance of Scapholebris, $17=$ occurrence of Scapholebris, $18=$ oxygen content. 

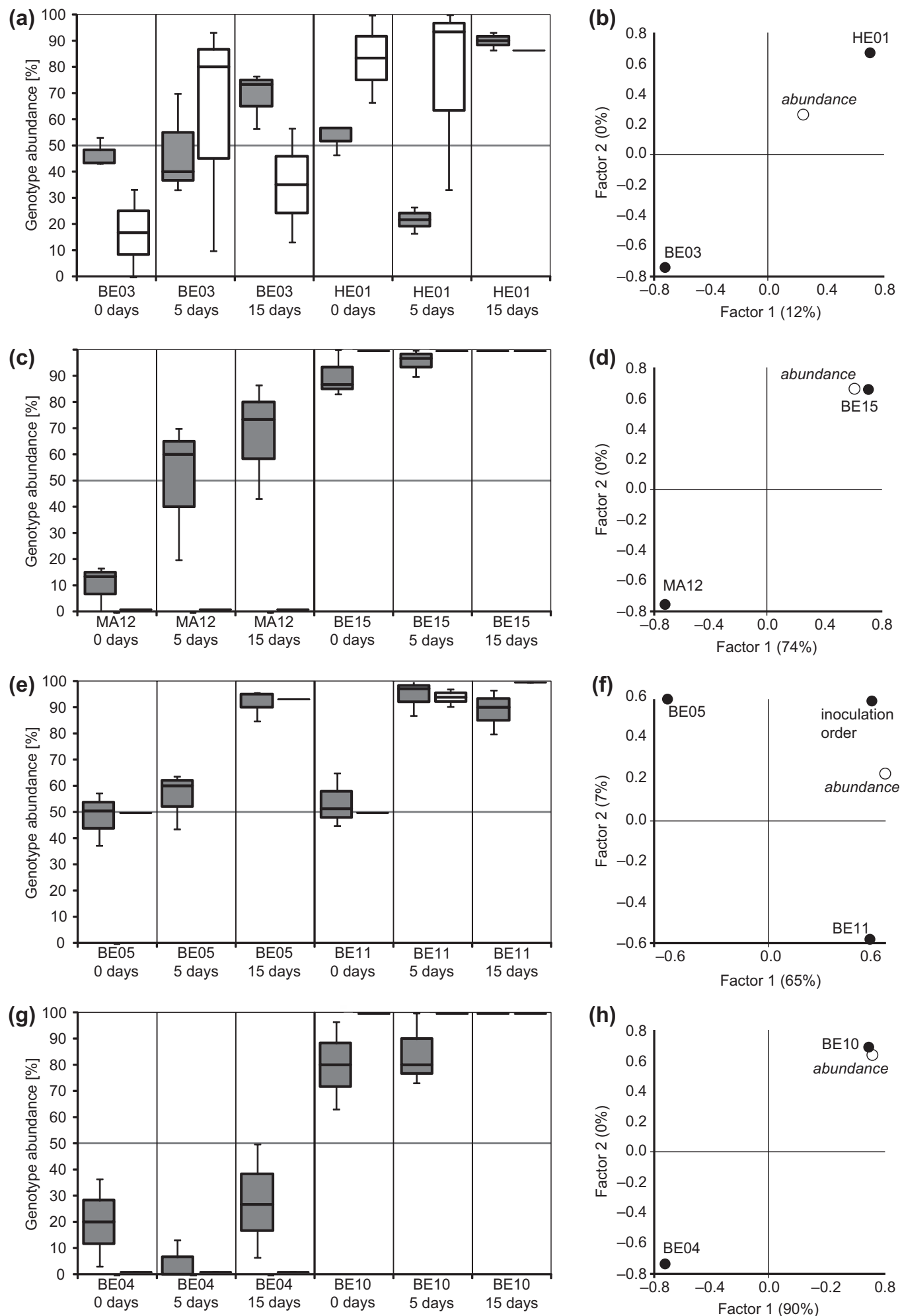

Figure 2. Box plots of all data showing abundances for each founder genotype and inoculation order $(0$ days $=$ inoculated simultaneously with combination partner; 5 days = inoculated 5 days before the second corresponding genotype; 15 days = inoculated 15 days before the second corresponding genotype) of all genotype combinations (2a: BE03/HE01; 2c: MA12/BE15; 2e: BE05/BE11; 2g: BE04/BE10) for both sampling events (six weeks: first box plot of each genotype and treatment, grey; nine months: second box plot of each genotype and treatment, white). The abundances of the second (invading) genotypes are not shown because they are equivalent to the difference between the genotype abundance (\%) of the founder genotype and 100 percent. PLS regression plots of the two principal components with $\mathrm{x}$ and y loadings for each single genotype combination (2b, 2d, 2f, and $2 \mathrm{~h}$ ). Only significant $\mathrm{x}$-variables (black filled circles) explaining the y-variable genotype abundance (abundance, open circles) are shown. Full cross validation for each genotype combination: BE03/HE01 (2b; $\left.\mathrm{R}^{2}=0.07, \mathrm{RSME}=28.82\right), \mathrm{MA12} / \mathrm{BE} 15\left(2 \mathrm{~d} ; \mathrm{R}^{2}=0.90, \mathrm{RSME}=14.74\right), \mathrm{BE} 05 / \mathrm{BE} 11\left(2 \mathrm{f} ; \mathrm{R}^{2}=0.73, \mathrm{RSME}=13.24\right), \mathrm{BE} 04 / \mathrm{BE} 10(2 \mathrm{~h}$ $\left.\mathrm{R}^{2}=0.74, \mathrm{RSME}=23.55\right)$. 
was the only other variable that significantly contributed to genotype abundance according to PLS regression (Fig. 1), even though over all combinations it seemed to have less influence than fitness. To obtain more detailed insight into the patterns, we analysed each genotype combination separately (Fig. 2).

For genotypes BE03 and HE01, the pattern was not consistent over sampling periods (Fig. 2a-b). When clone BE03 was inoculated first, a five days advantage did not result in an increase in genotype abundance, but an advantage of 15 days resulted in a detectable effect after six weeks. After nine months, the pattern was different, with clone BE03 as the inferior partner in this clone pair except when it had an advantage of five days (although the variance was extremely high). When clone HE01 was inoculated first, an increase in abundance (founder effect) was observed in the case of a 15 days delay before the invading genotype was inoculated, which was maintained after nine months. In the case of a five days advantage, the pattern was reversed after six weeks (decrease in abundance), but after nine months a founder effect was observed. Overall, a 15 days advantage resulted in an observable founder effect.

In the competition trials with genotypes MA12 and $\mathrm{BE} 15$, giving a time advantage to the weaker competitor (clone MA12) resulted in a compensation of the difference in competitive strength after six weeks in the case of a five days advantage and in a slight dominance of this genotype in the case of a 15 days advantage (Fig. 2c). However, after nine months, the competitively inferior clone was not detected irrespective of inoculation order.

In the trial including genotypes BE05 and BE11, which showed similar competitive strength when inoculated simultaneously, we observed a clear-cut founder effect after six weeks if either genotype had a time advantage of 15 days
(Fig. 2e). After nine months, this pattern was maintained. PLS regression indicates an equal contribution of fitness and founder effects to the genotype abundances observed in this genotype pair (Fig. 2f).

Finally, in the competition between clones BE04 and $\mathrm{BE} 10$, genotype BE10 was always the superior one and even a 15 days advantage of the inferior clone resulted only in a slight increase in abundance of BE04 (Fig. 2g). After nine months, the inferior clone was not detected in any of the experimental container.

Although the fitness of genotypes is a strong contributor to genotype abundances in a population, we observed an increase in genotype abundance in almost all cases due to inoculation order (Fig. 3).

\section{Discussion}

\section{Parameters impacting the abundances of genotypes during population build-up}

Our aim was to determine to which extent the frequencies of different genotypes in a founder population are impacted by the succession sequence (founder effects) and by the differential fitness of those genotypes, respectively. That succession of individuals arriving at an unoccupied habitat may have severe consequences for population genetic structure was, for example, demonstrated for island populations as well as for populations at expanding frontiers (Waters et al. 2013). The build-up of new populations is often dominated by founder effects and genetic drift and the progeny of individuals first establishing in an unoccupied habitat are most likely to dominate the population gene pool (Ventura et al. 2014).

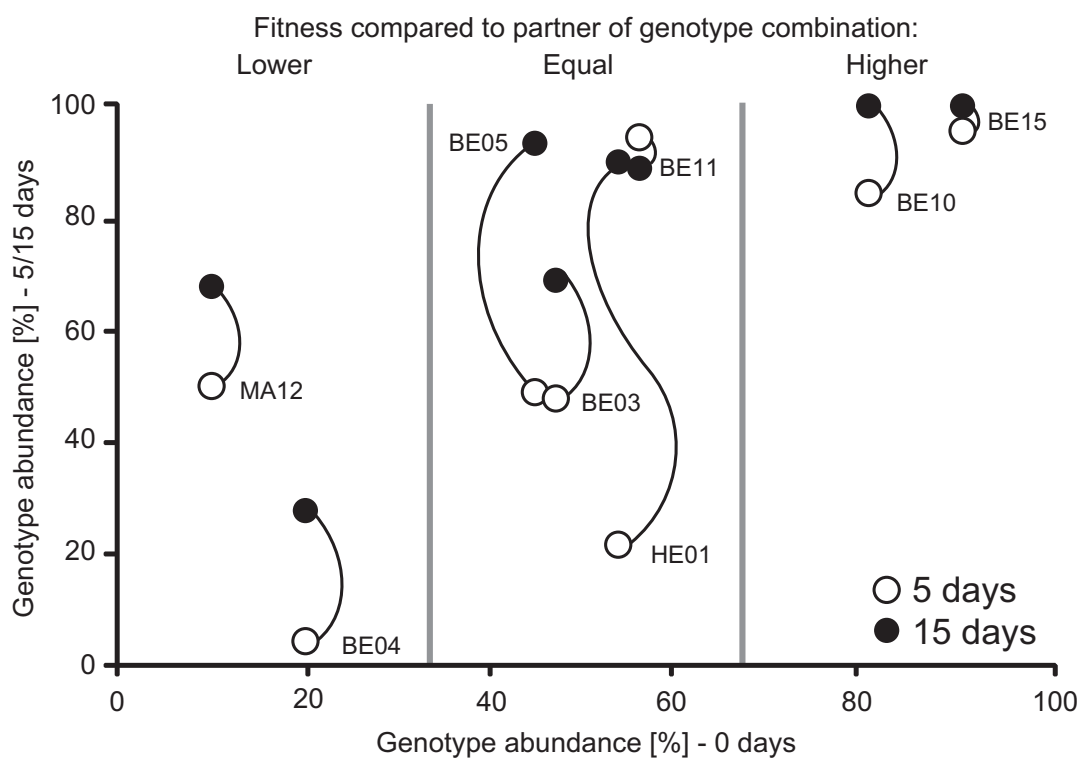

Figure 3. Relationship between fitness of each genotype (derived from the 0 days treatments) and the influence of inoculation order on the abundance of genotypes based on the data of the six weeks sampling. Clonal strength is shown on the $\mathrm{x}$-axis as mean relative abundance of each genotype if inoculated simultaneously with their competition partner. If abundance of both genotypes of the same combination did not differ significantly (based on 0 days treatment; see G-statistics in Results section for more information) then those genotypes were considered to show equal fitness. However, if genotypes showed significant differences in abundance then they were considered to have either lower or higher fitness. Founder effects are shown on the y-axis as relative abundance of each genotype, either with a time advantage of 5 (open circles) or 15 days (closed circles). 
In three of four clonal combinations we found evidence for founder effects, as differences in genotype abundances were observed related to the sequence of inoculation (with a time lag of 5 or 15 days) after approximately six weeks (four generations) of population build-up (Fig. 1b, 2). Our results are in line with the few other experimental studies dealing with founder effects during population build-up (Fukami et al. 2007, van Gremberghe et al. 2009). For example, van Gremberghe et al. (2009) recorded founder effects among different strains of the cyanobacterium Microcystis using an experimental approach over a time period encompassing several generations, while Fukami et al. (2007) detected differences in diversification of bacterial populations of different Pseudomonas fluorescens strains due to founder effects. In general, we observed that founder effects are much more pronounced if the first colonizers had a 15 compared to a 5 days time advantage (Fig. 3).

In some cases (combination of BE05 and BE11 both ways) the patterns after nine months were even reinforced compared to that after six weeks, suggesting that the founder effect was not a temporary advantage but rather a longlasting effect. However, in other clonal trials (e.g. MA12 and BE15), the initial founder effect was overruled by the competitively superior genotype.

Overall, we observed a strong interdependence of differences in competitive ability and the strength of founder effects (Fig. 3). We observed clear-cut and reciprocal founder effects in the trials with two clones that were competitively similar to each other (e.g. clones BE05 and BE11 and to a lesser extent clones BE03 and HE01), while founder effects were unclear or transient in the trials with combinations of clones that differed strongly in competitive strength. A numerical advantage through inoculation order as applied in our experiment was not sufficient to overrule strong differences in fitness among genotypes. In other words: the stronger the fitness differences among clones, the more deterministic their relative abundances are, and the less they are dependent on inoculation order.

Our results indicate that if fitness differences are not strong, founder effects exhibit a higher impact and also result in longer-lasting effects than when fitness differences are pronounced. When clones are competitively similar or slightly inferior to their competitor, founder effects are most pronounced. For clones that are strongly inferior competitors, an initial numerical advantage may very rapidly be annihilated and any founder effect may be lost. Clone BE04 in our trials is an example for this phenomenon.

\section{Genotype fitness and founder effects shaping population genetic structure}

Population genetic analyses of cyclically parthenogenetic zooplankton, like Daphnia, often report high levels of among-population genetic differentiation and endemism (Innes 1991, Gómez and Carvalho 2000, Gouws and Stewart 2007, Muñoz et al. 2008, Thielsch et al. 2009, Xu et al. 2009), suggesting low levels of ongoing gene flow. Still, freshwater organisms have been shown to exhibit a relatively high dispersal capacity thanks to the production of dormant stages that can be passively transported via waterfowl and wind (Havel and Shurin 2004). De Meester et al. (2002) have proposed the monopolization hypothesis as an explanation for this paradox between low gene flow and high dispersal capacity. This hypothesis states that the observed patterns of high among-population genetic differentiation may be explained by a combination of stochastic and selectiondriven processes. After historical colonisation from one or a few propagules, the rapid population growth rate combined with local genetic adaptation reduces effective gene flow by lowering the establishment success of late arriving genotypes (De Meester et al. 2002), resulting in an enhanced founder effect. Our study provides support for this hypothesis by showing that, if fitness differences among clones are not strong, founder effects being caused by the order in which genotypes arrive in a target habitat can be important and result in long-lasting changes in genetic composition among populations. As local genetic adaptation increases fitness of residents compared to the average invading genotype (which was not studied here, as we worked with clones), one would expect that the founder effects we observed in mesocosms would be enhanced in natural communities. Importantly, the time span over which we observed founder effects is very short: in some cases a long-lasting (nine months) effect is present already when a time advantage of 5 days is given, whereas a time advantage of 15 days resulted in founder effects in most of our experimental populations. This is in line with earlier results at the interspecific level, in which similarly strong priority effects were observed in a study with different species of large-bodied cladocerans (Louette et al. 2007).

The large impact of even a short time advantage leads to the expectation that in nature a time difference of a few months or years is likely to have an important effect, especially given that local populations produce a large dormant egg bank (De Meester et al. 2002). Juveniles hatch from egg banks when conditions become favourable. Given the vast size of most egg banks (Brendonck and De Meester 2003), invasion by new genotypes may only be possible during a very small time window each year during which population densities or genetic diversity are relatively low. Ortells et al. (2012) studied the population genetic structure of D. magna over five growing seasons and detected a population with high numbers of invading clones for the first growing season but little evidence for further successful invasions during the following growing seasons. New alleles were detected at two studied microsatellite loci, but the frequencies were marginal. This illustrates that founder effects as observed in our experimental study may be an important factor in shaping genetic structure also in natural populations.

Importantly, though, our results also show the limitations of founder effects, which are dictated by the differences in fitness among clones. Our results indeed indicate that a superior genotype may overrule founder effects, consistent with observations by Ebert et al. (2002). This highlights the importance of local genetic adaptation in contributing to long-standing founder effects in nature, as local genetic adaptation results in a decline in the probability that a genotype of higher fitness than the residents will arrive in a habitat.

\section{Conclusion}

Our results identified that two different processes interact strongly in determining population genetic structure 
during population build-up in the water flea Daphnia. First, founder effects play an important role, with an advantage of starting a few days earlier being sufficient for a genotype to dominate a population. Although we only compared two time lags, our results indicate that the more time a first colonist is given to grow before an invading genotype reaches the population, the stronger the impact of this advantage and the more likely that it becomes permanent. Even if we ignore differences in arrival time of genotypes within a given growing season, our results suggest that genotypes which colonize a new habitat in the first year may have a strong and potentially long-lasting advantage over later arriving genotypes, which may result in strong and persistent founder effects if the number of colonists each year is relatively low (Louette et al. 2007, Ortells et al. 2012, 2014). Second, it is well established that Daphnia genotypes can strongly differ in fitness in a given habitat, and clonal selection may be very effective. Our results confirm this, and indicate that founder effects can be overwhelmed by intrinsic fitness differences among clones. During the initial stages of habitat colonization, these fitness differences may reduce the impact of founder events and may change their impact on population genetic structure by changing the identity of the clones that dominate in a habitat. As a population gets established and genetically adapts to local environmental conditions, however, we expect that fitness differences will tend to be biased in favour of residents and may as such stabilize the patterns of genetic differentiation resulting from founder events (De Meester et al. 2002). This at least is expected when environmental conditions remain stable. It is conceivable that invading genotypes may have higher fitness in the case when environmental conditions change. The degree to which this occurs needs further study, as it depends on the fitness profiles of genotypes in the regional genotype pool as well as on evolutionary potential of local populations, for instance, their capacity to rapidly track environmental change. Most studies on Daphnia suggest that rapid genetic tracking of environmental change is possible (Van Doorslaer et al. 2009, Jansen et al. 2011), and Van Doorslaer et al. (2009) did also show that local adaptation may reduce establishment success of pre-adapted genotypes from the region. Yet, the continuous adaptation of parasites to local populations (Decaestecker et al. 2007) may challenge the adaptive superiority of the residents (Ebert et al. 2002).

Acknowledgements - This project was supported by the German Science Foundation (DFG) project SCHW 830/6-1, EuroCORES/ EuroDIVERSITY project BIOPOOL (European Science Foundation), the Biodiversity and Climate Research Centre Frankfurt am Main (BiKF; "LOEWE - Landes-Offensive zur Entwicklung Wissenschaftlich-ökonomischer Exzellenz" of Hesse's Ministry of Higher Education, Research, and the Arts), and KULeuven research fund projects GOA/2008/06 and PF/2010/07. Furthermore, we thank the Otto Stern School for awarding a travel grant to present part of this work at the VIIIth International Symposium on Cladocera in Aguascalientes, Mexico. We are thankful to Stephanie Domaschke, Timm Haun and Maike Herrmann for helping with sample preparation and genotype identification, and to members from the lab of Luc De Meester who helped with setting up and monitoring the mesocosms. We are very grateful to Steven Declerck for advice and support with experimental design as well as to Constanze Buhk for guidance on PLS regression analyses.

\section{References}

Abdi, H. 2007. Partial least square regression. - In: Salkind, N. (ed.), Encyclopedia of measurement and statistics. Sage, pp. 741-745.

Allen, M. R. 2007. Measuring and modeling dispersal of adult zooplankton. - Oecologia 153: 135-143.

Bieri, A. P. S. et al. 2009. Aphid genotypes vary in their response to the presence of fungal endosymbionts in host plants. $-\mathrm{J}$. Evol. Biol. 22: 1775-1780.

Boileau, M. G. et al. 1992. Non-equilibrium gene frequency divergence: persistent founder effects in natural populations. - J. Evol. Biol. 5: 25-39.

Brede, N. et al. 2006. Microsatellite markers for European Daphnia. - Mol. Ecol. Notes 6: 536-539.

Brendonck, L. and De Meester, L. 2003. Egg banks in freshwater zooplankton: evolutionary and ecological archives in the sediment. - Hydrobiologia 491: 65-84.

Campillo, S. et al. 2009. Selection on life-history traits and genetic population divergence in rotifers. - J. Evol. Biol. 22: 2542-2553.

Carrascal, L. M. et al. 2009. Partial least squares regression as an alternative to current regression methods used in ecology. - Oikos 118: 681-690.

De Meester, L. et al. 2002. The monopolization hypothesis and the dispersal-gene flow paradox in aquatic organisms. - Acta Oecol. 23: 121-135.

Decaestecker, E. et al. 2007. Host-parasite 'Red Queen' dynamics archived in pond sediment. - Nature 450: 870-873.

Ebert, D. et al. 2002. A selective advantage to immigrant genes in a Daphnia metapopulation. - Science 295: 485-488.

Fukami, T. et al. 2007. Immigration history controls diversification in experimental adaptive radiation. - Nature 446: 436-439.

Gómez, A. and Carvalho, G. R. 2000. Sex, parthenogenesis and genetic structure of rotifers: microsatellite analysis of contemporary and resting egg bank populations. - Mol. Ecol. 9: 203-214.

Gouws, G. and Stewart, B. A. 2007. From genetic structure to wetland conservation: a freshwater isopod Paramphisopus palustris (Phreatoicidea : Amphisopidae) from the Swan Coastal Plain, Western Australia. - Hydrobiologia 589: 249-263.

Havel, J. E. and Shurin, J. B. 2004. Mechanisms, effects, and scales of dispersal in freshwater zooplankton. - Limnol. Oceanogr. 49: 1229-1238.

Hewitt, G. M. and Ibrahim, K. M. 2001. Inferring glacial refugia and historical migrations with molecular phylogenies. - In: Silvertown, J. and Antonovics, J. (eds), Integrating ecology and evolution in a spatial context. Blackwell, pp. 271-294.

Hood, G. M. 2009. PopTools ver. 3.1.1. - <www.cse.csiro.au/ poptools $>$.

Innes, D. J. 1991. Geographic patterns of genetic differentiation among sexual populations of Daphnia pulex. - Can. J. Zool. 69: 995-1003.

Jansen, M. et al. 2011. Collateral damage: rapid exposure-induced evolution of pesticide resistance leads to increased susceptibility to parasites. - Evolution 65: 2681-2691.

Klüttgen, B. et al. 1994. ADaM, an artificial freshwater for the culture of zooplankton. - Water Res. 28: 743-746.

Louette, G. and De Meester, L. 2005. High dispersal capacity of cladoceran zooplankton in newly founded communities. - Ecology 86: 353-359.

Louette, G. et al. 2007. The founding mothers: the genetic structure of newly established Daphnia populations. - Oikos 116: 728-741.

Macháček, J. and Sed'a, J. 2007. Life history response of Daphnia galeata to heterogeneous conditions within a reservoir as determined in a cross-designed laboratory experiment. - Aquat. Ecol. 41: 55-66. 
Morin, P. J. 1999. Community ecology. - Blackwell.

Muńoz, J. et al. 2008. Phylogeography and local endemism of the native Mediterranean brine shrimp Artemia salina (Branchiopoda : Anostraca). - Mol. Ecol. 17: 3160-3177.

Ortells, R. et al. 2012. Colonization in action: genetic characteristics of Daphnia magna Strauss (Crustacea, Anomopoda) in two recently restored ponds. - Hydrobiologia 689: 37-49.

Ortells, R. et al. 2014. Colonization of Daphnia magna in a newly created pond: founder effects and secondary immigrants. Hydrobiologia 723: 167-179.

Schwenk, K. et al. 1998. Genetic markers, genealogies and biogeographic patterns in the cladocera. - Aquat. Ecol. 32: 37-51.

Symonds, V. V. and Lloyd, A. M. 2004. A simple and inexpensive method for producing fluorescently labelled size standard. Mol. Ecol. Notes 4: 768-771.

Thielsch, A. et al. 2009. Contribution of cyclic parthenogenesis and colonization history to population structure in Daphnia. - Mol. Ecol. 18: 1616-1628.

Van Doorslaer, W. et al. 2009. Local adaptation to higher temperatures reduces immigration success of genotypes from a warmer region in the water flea Daphnia. - Global Change Biol. 15: 3046-3055.

Supplementary material (available online as Appendix oik.01575 at $<$ www.oikosjournal.org/readers/appendix $>$ ). Appendix 1. van Gremberghe, I. et al. 2009. Priority effects in experimental populations of the cyanobacterium Microcystis. - Environ. Microbiol. 11: 2564-2573.

Ventura, M. et al. 2014. Local and regional founder effects in lake zooplankton persist after thousands of years despite high dispersal potential. - Mol. Ecol. 23: 1014-1027.

Waters, J. M. et al. 2013. Founder takes all: density-dependent processes structure biodversity. - Trends Ecol. Evol. 28: 78-85.

Wold, H. 1975. PLS path models with latent variables: the nipals approach. - In: Blalock, H. M. et al. (eds), Quantative sociology: international perspectives on mathematical and statistical modeling. Academic Press, pp. 391-420.

Wold, S. et al. 2001. PLS-regression: a basic tool of chemometrics. - Chemometr. Intell. Lab. 58: 109-130.

$\mathrm{Xu}, \mathrm{S}$. et al. 2009. The noncosmopolitanism paradigm of freshwater zooplankton: Insights from the global phylogeography of the predatory cladoceran Polyphemus pediculus (Linnaeus, 1761) (Crustacea, Onychopoda). - Mol. Ecol. 18: 5161-5179.

Zaffagnini, F. 1987. Reproduction in Daphnia. - In: Peters, R. H. and R. de Bernardi (eds), Memorie dell'Istituto italiano di Idrobiologie: Daphnia. Consiglio Nazionale delle Ricerche, pp. 502 . 